AN UNUSUAL CASE OF FAMILIAL FCOD

\author{
Saravana Selvam S1, C. S. C. Satish Kumar², Sathiya Jeeva ${ }^{3}$
}

${ }^{1}$ Senior Lecturer, Department of Oral Medicine and Radiology, Thai Moogambigai Dental College and Hospital, Chennai, Tamilnadu, India.

2Senior Lecturer, Department of Oral and Maxillofacial Surgery, Thai Moogambigai Dental College and Hospital, Chennai, Tamilnadu, India.

${ }^{3}$ Reader, Department of Oral Pathology, Thai Moogambigai Dental College and Hospital, Chennai, Tamilnadu, India.

HOW TO CITE THIS ARTICLE: Selvam SS, Kumar CSCS, Jeeva S. An unusual case of familial FCOD. J. Evolution Med. Dent. Sci. 2018;7(33):3721-3723, DOI: 10.14260/jemds/2018/835 \section{PRESENTATION OF CASE}

A 55-year-old female patient came to our department with a chief complaint of routine dental check-up and wants to get an age certificate for her company where she works. Intraoral examination revealed calculus and stains were moderate and missing tooth in relation to 15 . Radiological investigation was done in which orthopantomograph revealed well-defined radio-opaque areas evident in relation to the periapical aspect of 36 regions measuring about $1 \times 1 \mathrm{~cm}$ in dimension. Then advanced imaging modality CBCT was taken to know the exact nature and extent, which revealed a small welldefined radio-opaque lesion in the periapical region of 37. Mild inter-dental bone loss was evident in relation to 36,37 region. Only periodic follow-up and good oral care were advised to the patient.

\section{Case-2}

A 32-year-old female patient came to our department with a chief complaint of routine dental check-up and wants to get an age certificate for her company where she works. Intraoral examination revealed missing teeth in relation to 26,46 . Radiological investigation was done in which intraoral periapical radiograph revealed mixed radiolucent radioopaque lesion in the periapical aspect of 46, 47 measuring about $2 \times 1 \mathrm{~cm}$ in dimension. Orthopantomograph revealed mixed radiolucent radio-opaque lesion in the periapical aspect of 36, 37 and 46, 47. Then advanced imaging modality CBCT was taken to know the exact nature and extent, which revealed mixed radio-opaque radiolucent lesion in periapical aspect of 36, 37 and 46, 47 with mild cortical expansion bilaterally in the molar regions. Only periodic follow-up and good oral care were advised to the patient.

The most interesting phenomenon in these two patients was that they were both mother and daughter, and both having periapical radiopacities. Familial case of FCOD is extremely rare and as per our knowledge only one familial case has been reported in India.

'Financial or Other Competing Interest': None.

Submission 04-07-2018, Peer Review 28-07-2018,

Acceptance 03-08-2018, Published 13-08-2018.

Corresponding Author:

Dr. Saravana Selvam S,

Senior Lecturer,

Department of Oral Medicine and Radiology,

Thai Moogambigai Dental College and Hospital,

Chennai, Tamilnadu, India.

E-mail: drsaravana2512@gmail.com

DOI: $10.14260 /$ jemds $/ 2018 / 835$

(c) $($ )

\section{CLINICAL DIAGNOSIS}

The high incidence and broad-spectrum of conditions causing periapical radiolucency and radiopacity make it imperative that all dental clinicians acquire a broad and comprehensive working knowledge as some of these may present as innocent anatomic variations, whereas others are a result of benign or malignant conditions. Fibro-osseous lesions (FOL) are a poorly defined group of lesions affecting the jaws and craniofacial bones. All are characterised by the replacement of bone by cellular fibrous tissue containing foci of mineralisation that vary in amount and appearance. ${ }^{1}$ The term was first described by Melrose et al in 1976. The word florid was introduced to describe the widespread, extensive manifestation of the disease. ${ }^{2}$ The disease has a tendency for bilateral occurrence often symmetrically. ${ }^{3}$ Focal cementoosseous dysplasia (FCOD) in the tooth bearing areas of the jaws is an asymptomatic benign condition, belonging to the spectrum of fibro-osseous lesions. ${ }^{1}$ The aetiology and pathogenesis of FCOD is unknown and this lesion is considered to be a reactive or a dysplastic process in the periapical tissues. Usually, it affects two or more mandibular anterior teeth and the radiographic appearance varies depending on the state of development. In rare cases, the lesion may affect only one tooth mimicking an apical granuloma or a cyst. 4

FCOD is a type of sclerosing lesion characterised by multiple exuberant lobulated densely opaque masses restricted to alveolar process in either or both jaws. When the lesions are large, jaw expansion may be noted with dull pain or drainage in affected area. It should be differentiated from chronic sclerosing, osteomyelitis, condensing osteitis, enostosis and odontoma. Here, we present a rare case of a familial FCOD noted in both mom and daughter through radiographic investigations.

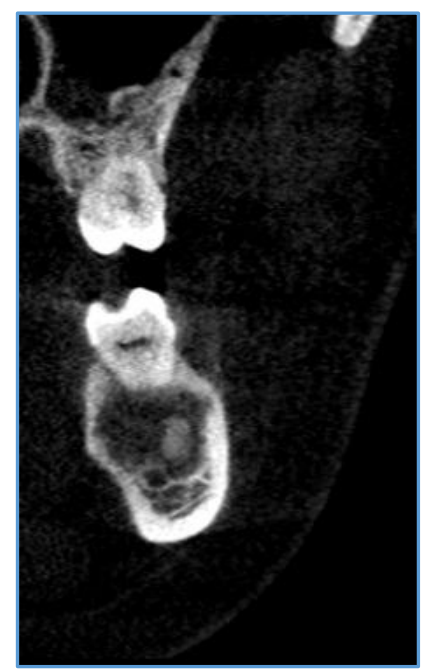

Case 1. CBCT of Left Posterior Mandibular Region 


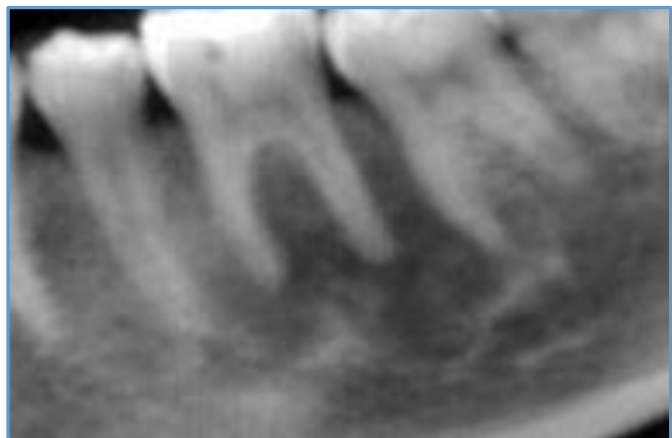

Case 2. Left Posterior Mandibular region showing Mixed Radiolucent Radiopaque Structure

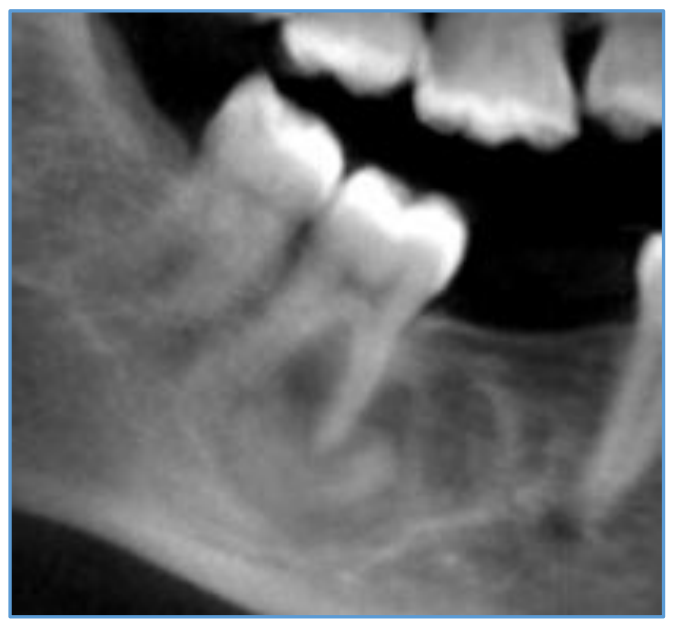

Case 2. Right Posterior Mandibular region showing Mixed Radiolucent Radiopaque Structure

\section{DISCUSSION}

"Benign fibro-osseous lesion" is a well-known term that encompasses a wide range of conditions, the diagnoses of which are often challenging. The differential diagnosis of cemento-osseous dysplasia (COD) may include several possible entities, which must consider the stage of a lesion's development and the possible co-existence with associated entities like diffuse osteomyelitis or simple bone cyst. Consequently, there have been many reports of misdiagnoses and mismanagement of COD cases. 5

The important role of the oral and maxillofacial radiologist in the diagnosis of a "fibro-osseous lesion" derives from often similarly-appearing histological presentations. Consequently, the radiographic appearances may be of value in differentiating cemento-osseous dysplasias from other lesions that may also be categorised as being of a "fibroosseous" nature.

New cases of cemento-osseous dysplasias are diagnosed based on radiographic features alone and a correct diagnosis may prevent unnecessary surgery. Therefore, the purpose of this study was to focus on the radiographic aspects of the cemento-osseous dysplasias and to determine if an association can be made between one or more characteristic radiographic features.

The FCOD's predilection for females is a significant feature shared only with the FCOD. Kawai and co-authors reported that very few Japanese males affected with these lesions were older than the females. They suggest that CODs represent a dysplastic process related to hormonal imbalance, which influences bone remodelling. ${ }^{6}$
Unfortunately, this hypothesis remains merely until clearer evidence is obtained by full gynaecological histories and hormonal testing of larger groups of patients who have been followed up over a long period. In our case, both patients were female which was correlating with other authors.

In a study of 54 subjects with COD, Kawai et al reported that the mean age of the five male subjects was 64.4 years compared to the mean age of females of 49.4 years. These authors also related the prevalence of COD in women to their hypothesis that "COD lesions represent a dysplastic process related to hormonal imbalance, which influences bone remodeling."6 However, the absence of any hormonal testing of this group of subjects makes this association unsupportable. Whereas in our case, the correlation was done between mother and daughter whose ages were 46 and 30 respectively.

According to the archived reports, most COD cases were solitary and unilateral $(51.7 \%$ and $77.1 \%$, respectively). The majority of the CODs (81.4\%) were reported in the mandible and $44.9 \%$ were related to the posterior teeth only. $6,7,8,9$ The jaw distribution of COD reported in the literature varied according to the subtype of COD being studied. In periapical (or focal) COD case reports or series, the lesions were mostly solitary, whereas with florid COD cases bilateral occurrence increased. In our case one was unilateral and another was bilateral and both the cases were confined to the posterior part of the mandible.

Few studies examined the periphery of COD lesions. Su et al ${ }^{1}$ reported that $53 \%$ of 142 cases of focal COD were welldefined. In their study, radiographs included panoramic or periapical radiographs and were examined independently by two of the authors. On the other hand, Summerlin and Tomich ${ }^{10}$ reported that the peripheries of most of their 221 cases of focal COD were ill-defined. Later, however in their discussion, the authors described the majority of entities as being "fairly well-defined." The authors failed to report frequencies or proportions of radiographic features, did not mention the radiographic images used or how they were analysed. In both our cases, it was well-defined periphery.

With respect to internal structure most COD cases were reported to be in the mixed radiolucent/ radiopaque stage and the internal radiopacities were described as being either one or a mixture of cementum-like ground glass or "Pagetoid" (a term used mostly in the description of extensive radiopacities in florid COD cases). In our case, it was found to be in mixed radiolucent/ radiopaque stage. ${ }^{1,6}$

A point of controversy is the presence or absence of jaw expansion caused by the COD lesion. Few published pathology-based reviews describe COD lesions as being nonexpansile.10,11 In other reports where occlusal radiographs were examined, a slight expansion of at least one of the mandibular cortical plates was reported with intact, thinned cortices. In our case, there was no expansion of the mandible noted.

Oral radiologists utilised six radiographic features of COD that when identified were associated more strongly with a correct interpretation of COD. The presence of a well-defined periphery, the presence of a radiolucent rim or border, the presence of mixed radiolucencies with radiopacities, bilateral distribution of lesions, involvement of anterior and posterior teeth and the absence of non-concentric bony expansion. Our case satisfied all the six radiographic features and guided in building the diagnosis of FCOD. 
The systematic evaluation of radiographic features (Location, periphery, internal structure and effects on the surrounding structures) of 37 cases of COD presented to six clinicians represents the largest objective radiology-based study till date. Furthermore, the author found significant differences in the abilities of oral radiologists and the general dentists to correctly interpret COD and to distinguish it from other similarly appearing entities. Based on radiographs only, the oral radiologists were able to identify several radiographic features that were deemed characteristic of COD and accordingly were able to correctly differentiate the majority of COD cases from dense bone island, cementoblastoma, cemento-ossifying fibroma, fibrous dysplasia, complex odontoma and sclerosing osteitis.

A familial correlation of FCOD has been less reported and to our knowledge only one case has been recorded in India. Our case had a familial similarity, which had both mother and daughter involved which was considered rare in India based on literature.

Treatment of FCOD varies from case to case. Complete resection is generally impractical, as lesion almost always occupied the entire mandible and maxilla. In asymptomatic patient, it is wise to keep the patient under observation without surgical intervention. If required a remodelling resection is recommended for aesthetic reasons. 12

The management of symptomatic patients is more difficult, because chronic inflammation and infection develop within these densely mineralised tissues. ${ }^{13}$ Antibiotics also have poor penetration in these areas and are not generally effective. In our case, mother and daughter were asymptomatic and both of them were kept under observation and were asked to report to the department every 6 months for a periodic review.

\section{FINAL DIAGNOSIS}

Focal cemento-osseous dysplasia is a benign, non-neoplastic, self-limiting disease. Nominal number of cases has been reported in the modern literature. FCOD presents with no symptoms and found incidentally on radiographs. In symptomatic patients, conservative approaches such as broad-spectrum antibiotics along with periodic follow-up is requisite and in asymptomatic individuals only periodic follow-up and good oral care is advised. Surgical intervention of concerned bone is required only for cosmetic concerned individuals. Although, clinical and radiographic findings were adequate to ascertain the diagnosis, in our cases histopathological investigation was not done because both our patients were asymptomatic and surgical intervention was not required.

\section{REFERENCES}

[1] Summerlin DJ, Tomich CE. Focal cemento osseous dysplasia - a clinical pathological study of 221 cases. Oral Surgery Oral Medicine and Oral Pathology 1994;78(5):611-20.

[2] Melrose RJ, Abrams AM, Mills BG. Florid osseous dysplasia. A clinical pathologic study of thirty- four cases. Oral Surgery Oral Medicine and Oral Pathology 1976;41(1):62-82.

[3] Waldron CA. Fibro-osseous lesions of the jaws. Journal of Oral and Maxillofacial Surgery 1985;43(4):249-62.

[4] Forman GH. Periapical Cemental Dysplasia resembling apical granulomata and radicular cysts. British dental Journal 1975;138(1):22-4.

[5] Chadwick JW, Alsufyani NA, Lam EW. The clinical and radiographic features of solitary and cemento-osseous dysplasia associated simple bone cysts. Dentomaxillofac Radiol 2011;40(4):230-5.

[6] Su L, Weathers DR, Waldron CA. Distinguishing features of focal cemento-osseous dysplasia and cemento-ossifying fibromas. II. A clinical and radiologic spectrum of 316 cases. Oral Surg Oral Med Oral Pathol Oral Radiol Endod 1997;84(5):540-9.

[7] Ogunsalu C, Miles D. Cemento-osseous dysplasia in Jamaica: review of six cases. West Indian Med J 2005;54(4):264-7.

[8] Cho BH, Jung YH, Nah KS. The prevalence, clinical and radiographic characteristics of cemento-osseous dysplasia in Korea. Korean J Oral Maxillofac Radiol. 2007;37(4):185-9.

[9] Goncalves M, Pispico R, Alves Fde A, et al. Clinical, radiographic, biochemical and histological findings of florid cemento-osseous dysplasia and report of a case. Braz Dent J 2005;16(3):247-50.

[10] Brannon RB, Fowler CB. Benign fibro-osseous lesions: a review of current concepts. Adv Anat Pathol 2001;8(3):126-43.

[11] Eversole R, Su L, ElMofty S. Benign fibro-osseous lesions of the craniofacial complex: a review. Head Neck Pathol 2008;2(3):177-202.

[12] Miyake M, Nagahata S. Florid cemento-osseous dysplasia: report of a case. International Journal of Oral and Maxillofacial Surgery 1999;28(1):56-7.

[13] Pitak-Arnnop P, Dhanuthai K, Chaine A, et al. Florid osseous dysplasia: report of a case presenting acute cellulitis. Medicina Oral, Patol Oral Cirugia Bucal 2009;14(9):e461-e4. 